\section{P2-97 HOMOCYSTEINE LEVELS AND DIETARY PATTERNS AMONG YOUNG ADULTS FROM A BIRTH COHORT IN BRAZIL}

doi:10.1136/jech.2011.142976i.32

'D Gigante, ${ }^{*}{ }^{2} \mathrm{M}$ T Olinto, ${ }^{1} \mathrm{I}$ Oliveira, ${ }^{1} \mathrm{~B}$ Horta. ${ }^{1}$ Universidade Federal de Pelotas, Pelotas, Rio Grande do Sul, Brazil; ${ }^{2}$ UNISINOS, São Leopoldo, Rio Grande do Sul, Brazil

Introduction Hyperhomocysteinemia has been associated with ischaemic heart disease and stroke. Dietary intake of folate and other vitamins is a major determinant of blood homocysteine concentration. The objective of this study is to analyse the plasma homocysteine concentrations in young adults who have been followed since birth. In addition, homocysteine concentrations are presented in according to the main dietary patterns previously identified in this cohort.

Methods The 1982 Pelotas birth cohort included 5914 children who were born in three maternities in a city of Southern Brazil (Pelotas). In 2004-2005, members of this cohort were interviewed and blood was collected in 3827 subjects. Food frequency questionnaire was applied in the interview and three main dietary patterns were defined by principal component analysis: common Brazilian (CB), processed food (PF) and vegetable/fruit (VF). Serum levels of homocysteine were determined using immunoassay analyser.

Results The mean of homocysteine was $8.45 \pm 3.27 \mu \mathrm{mol} / 1$, and it was higher in men $(9.50 \pm 3.71 \mu \mathrm{mol} / \mathrm{l})$ than in women $(7.39 \pm 2.32 \mu \mathrm{mol} / \mathrm{l})$. Higher means of homocysteine were observed among subjects from the third tertile of intake for $\mathrm{CB}$ and PF dietary patterns. However, an inverse association was observed between levels of homocysteine and the tertiles of VF dietary pattern. The means of homocysteine were 8.73 \pm 3.36 , 8.44 \pm 3.38 , and $8.19 \pm 3.09 \mu \mathrm{mol} / 1$, respectively in the tertiles of VF dietary pattern $(\mathrm{p}<0.001)$.

Conclusion These findings suggest that homocysteine concentrations were lower among subjects who adhered to the healthy dietary pattern.

\section{P2-98 PRIOR PSYCHIATRIC HOSPITALISATION PREDICTS MORTALITY IN PATIENTS HOSPITALISED WITH NON- CARDIAC CHEST PAIN: A DATA LINKAGE STUDY BASED ON THE FULL SCOTTISH POPULATION (1991-2006)}

doi:10.1136/jech.2011.142976i.33

${ }^{1} \mathrm{M}$ Gillies, ${ }^{*} \mathrm{~K}$ MacTeague, ${ }^{1} \mathrm{P}$ Jhund, ${ }^{2} \mathrm{D}$ Batty, ${ }^{3} \mathrm{~J}$ Allardyce, ${ }^{1} \mathrm{P}$ Maclntyre, ${ }^{1} \mathrm{~K}$ Macintyre. ${ }^{1}$ University of Glasgow, Glasgow, UK; ${ }^{2}$ University College London, London, UK; ${ }^{3}$ Maastricht University, Maastricht, The Netherlands

Introduction Non-cardiac chest pain (NCCP) is considered a benign condition, associated with a low mortality rate. Contemporary population based studies describing outcomes in NCCP are lacking however. To our knowledge, the relationship between psychiatric disorders and survival in patients with NCCP has not been investigated. This data linkage study investigated case-fatality following a first hospitalisation for NCCP and examined the effect of a previous psychiatric hospitalisation on short-term all-cause and CVD specific mortality.

Methods A population-based retrospective cohort study of 159888 patients discharged from hospital in Scotland (1991-2006) following a first hospitalisation for NCCP using routinely collected hospital morbidity and mortality data. All-cause and cardiovascular disease (CVD) mortality at 1 year following hospitalisation was examined. Results $4.4 \%$ (3514) of men and 3.9\% (3136) of women with a first NCCP hospitalisation had a psychiatric hospitalisation in the 10 years preceding incident NCCP hospitalisation; those with a previous psychiatric hospitalisation were younger and more socioeconomically deprived (SED). Crude case-fatality at 1 year was higher in patients with a previous psychiatric hospitalisation compared to those without (men $6.3 \%$ vs $4.3 \%$; women $5.4 \%$ vs $3.6 \%$ ), in all age groups and all SED quintiles. Following adjustment (year of NCCP hospitalisation and SED), the hazard of all-cause and CVD-specific mortality at 1 year was higher in men and women with a previous psychiatric hospitalisation, and inversely related to age.

Conclusions Previous psychiatric hospitalisation should be considered in the risk stratification of patients discharged from hospital with a diagnosis of NCCP. Cardiovascular prevention strategies should target this hard to reach group.

\section{P2-99 TIME TRENDS IN FIRST AND SUBSEQUENT HOSPITALISATION FOR COPD IN SCOTLAND, 1991-2009}

doi:10.1136/jech.2011.142976i.34

M Gillies, ${ }^{*}$ J Lewsey, H Starkie, A Briggs, M Shepherd, K Maclntyre. University of Glasgow, Glasgow, UK

Introduction Chronic obstructive pulmonary disease (COPD) is a major cause of morbidity and mortality. Long-term trends describing the hospital burden of COPD are sparse.

Methods A retrospective cohort study using the Scottish Morbidity Record Scheme. First and subsequent hospitalisations for COPD in Scotland (1991-2009) were identified. Age standardised hospitalisation rates were calculated. Age and sex-specific trends in first and subsequent hospitalisation were modelled (Joinpoint regression)

Results There were 63996 first hospitalisations for COPD (1991-2009). Over time the rate of first hospitalisation fell in men and remained stable in women $>55$ years old, but rose in both men and women 35-54 years old. In total 64942 individuals contributed to 185200 readmissions. Readmission rates increased in men aged 35-54 years and $>75$ years old. A trend towards falling readmission rates in men aged $55-74$ years, in whom the burden of COPD is greatest, was observed. In women, rates of readmission increased in all age groups. However the rate of increase slowed in those aged 55-84 years in the latter period of the study.

Conclusions The hospital burden of COPD is high and driven by readmissions. Our data suggest that the COPD epidemic may be approaching a peak. However, incident hospitalisation for COPD in men and women 35-54 years old are increasing and as survival following an incident COPD hospitalisation improves and the population ages, the absolute number of hospital admission for COPD will increase. Alternates (cost-effective) models to hospital care are urgently required to meet this demand.

\section{P2-100 THE RELATIONSHIP BETWEEN POST TRAUMATIC STRESS DISORDER AND HYPERTENSION AMONG 105180 ASYLUM SEEKERS IN THE NETHERLANDS}

doi:10.1136/jech.2011.142976i.35

${ }^{1,2}$ S Goosen, ${ }^{*}{ }^{1} \mathrm{C}$ Agyemang, ${ }^{3} \mathrm{G}$ Ogedegbe. ${ }^{1}$ Department of Public Health, Academic Medical Centre, University of Amsterdam, Amsterdam, The Netherlands, ${ }^{2}$ Netherlands Association for Community Health Services, Utrecht, The Netherlands; ${ }^{3}$ Centre for Healthful Behavior Change, Division of General Internal Medicine, Department of Medicine, New York University School of Medicine, New York, USA

Objective To determine the relationship between post-traumatic stress disorder (PTSD) and hypertension among asylum seekers in the Netherlands.

Methods Data were obtained from the Dutch national electronic database of the Community Health Services for Asylum Seekers (MOA) from 2000 to 2008. Asylum seekers aged $\geq 18$ years at arrival in the reception facilities were included in this study $(\mathrm{N}=105180)$. The diagnosis of hypertension was coded according to the Dutch list of the International Classification of Primary Care, while PTSD was 